\title{
Targeting GH-1 Splicing as a Novel Pharmacological Strategy for Growth Hormone Deficiency Type II
}

Maria Consolata Miletta ${ }^{1}$, Christa E Flück ${ }^{1}$, Primus-E. Mullis ${ }^{1}$ 


\section{ABSTRACT (246 words)}

24 Isolated Growth Hormone Deficiency type II (IGHD II) is a rare genetic splicing disorder

25 characterized by reduced growth hormone $(\mathrm{GH})$ secretion and short stature. It is mainly caused

26 by autosomal dominant-negative mutations within the growth hormone gene $(G H-1)$ which result

27 in missplicing at the mRNA level and the subsequent loss of exon 3, producing the 17.5-kDa GH

28 isoform: a mutant and inactive GH protein that reduces the stability and the secretion of the 22-

$29 \mathrm{kDa} \mathrm{GH}$ isoform, the main biologically active $\mathrm{GH}$ form.

30 At present, patients suffering from IGHD II are treated with daily injections of recombinant

31 human $\mathrm{GH}(\mathrm{rhGH})$ in order to reach normal height. However, this type of replacement therapy,

32 although effective in terms of growth, does not prevent the toxic effects of the 17.5-kDa mutant

33 on the pituitary gland, which may eventually lead to other hormonal deficiencies. As the severity

34 of the disease inversely correlates with the $17.5-\mathrm{kDa} / 22-\mathrm{kDa}$ ratio, increasing the inclusion of

35 exon 3 is expected to ameliorate disease symptoms.

36 This review focuses on the recent advances in experimental and therapeutic strategies applicable

37 to treat IGHD II in clinical and preclinical contexts. Several avenues for alternative IGHD II 38 therapy will be discussed including the use of small interfering RNA (siRNA) and short hairpin 39 RNA (shRNA) constructs that specifically target the exon 3-deleted transcripts as well as the 40 application of histone deacetylase inhibitors (HDACi) and antisense oligonucleotides (AON) to 41 enhance full-lenght $G H-1$ transcription, correct $G H-1$ exon 3 splicing and manipulate GH 42 pathway.

44 Key Terms: Growth hormone, GH-1 gene, short stature, growth hormone deficiency type II, 45 splicing modulation. 
48 GROWTH HORMONE (GH) is a member of the somatotropin/prolactin family of hormones

49 and is considered of main importance for linear growth in childhood. Severe shortage or absence

50 of GH causes a condition called isolated growth hormone deficiency (IGHD).

51 IGHD is a rare disorder (the incidence of congenital IGHD varies between 1:4,000 - 10,000 live

52 births) and although it is most commonly sporadic, depending on the cohort screened, between

$53 \quad 3 \%-30 \%$ of cases are familial implicating genetic factors [1].

54 Newborns with a genetic aetiology of IGHD present a poor linear growth, which becomes 55 progressively retarded with age, and generally share many clinical features such as truncal 56 obesity, facial appearance younger than expected for their chronological age, delayed secondary 57 dentition and a high-pitched voice [2]. At the genetic level, familial IGHD has been usually 58 classified in four types depending on the inheritance pattern, as autosomal recessive (Type IA, 59 IB), autosomal dominant (Type II) or X-linked inherited (Type III) [2].

60 The most common genes implicated in the genetic aetiology of IGHD are those encoding GH $61(G H-1)$ and the receptor for GH releasing hormone $(G H R H R)$, while IGHD may be the only or 62 first presentation of mutations in genes encoding early (OTX2, HESX1, SOX2, SOX3) or late 63 (PROP1, POU1F1) transcription factors. Depending on the cohort studied and the criteria used 64 for defining IGHD, mutations in known genes are identified in almost $11 \%$ of IGHD patients, 65 with a higher prevalence in familial compared to sporadic IGHD (34\% vs 4\% respectively) [3].

66 In this review, we focus on IGHD II that is, in the majority of the cases, due to mutations 67 affecting splicing of the $G H-1$ gene [4-6]. These mutations result in skipping of exon 3 during 68 splicing and, therefore, production of a shorter GH variant: the 17.5-kDa GH isoform. At the 69 cellular level, the $17.5-\mathrm{kDa}$ GH isoform lacks the protein linker domain between the first two 
70 helices of $\mathrm{GH}$ and is therefore retained in the endoplasmic reticulum (ER). Basically, it acts as a

71 dominant negative isoform by blocking the secretion of the full-length $22-\mathrm{kDa} \mathrm{GH}$ isoform (wt-

$72 \mathrm{GH})[7,8]$ (Visual abstract).

73 For this reason, compared with healthy subjects, patients affected with IGHD II usually present 74 with decreased GH concentration although variations are observed depending on the specific 75 mutation affecting GH-1 splicing. In fact, clinical severity varies, and it has been shown to 76 correlate with the level of $17.5-\mathrm{kDa}$ GH isoform [9]. Several mechanisms are believed to cause 77 this dominant disorder, such as accumulation of toxic aggregates of mutant proteins, decreased 78 stability of $w t$-GH due to cellular responses induced by unfolded proteins or impaired maturation 79 of secretory vesicles (SVs). Nevertheless, a proper functional explanation for causing this 80 disorder remains unknown.

81 At present, children suffering from IGHD II are treated with costly daily injections of 82 recombinant human $\mathrm{GH}(\mathrm{rhGH})$ in order to reach normal height.

83 However, this type of approach, which is usually well tolerated and effective in terms of growth 84 and metabolism, can not replicate the normal, pulsatile pattern of GH secretion [10] and may not 85 totally prevent toxic effects of the $17.5-\mathrm{kDa}$ isoform on the pituitary gland, which can eventually 86 lead to other pituitary-derived hormonal deficiencies or to anterior pituitary hypoplasia [11]. 87 Moreover, children diagnosed with IGHD may require rhGH treatment for few years to lifelong 88 and the surveillance of the long term safety of rhGH treatment is still controversial [12].

89 Therefore, therapies that specifically target the damaging $17.5-\mathrm{kDa}$ GH isoform may be useful in 90 patients with $G H-1$ splicing defects.

91 This review summarizes the recent advances in experimental and therapeutic strategies applicable 92 to treat IGHD II and their possible future development as treatment. 


\section{2. The $G H-1$ gene cluster}

$94 \mathrm{GH}$ is a single-chain peptide of 191 amino acids produced and secreted mainly by the 95 somatotrophs of the anterior pituitary gland. It regulates many important processes such as 96 somatic growth, bone mineralization, sugar and lipid metabolism, protein synthesis and 97 stimulation of the immune system. GH production is, in turn, influenced by several factors 98 including sex, age, adiposity, sleep, stress, diet and exercise [13-17].

99 The gene encoding growth hormone $(\mathrm{GH}-\mathrm{l})$ is located on the long arm of chromosome 17 100 (17q22-24) within a cluster of five homologous genes, including CSH1 (chorionic 101 somatomammotropin hormone 1), $\mathrm{CSH} 2, \mathrm{CSHL1}(\mathrm{CSH}-$ like 1) and $G H 2$ (a GH variant expressed 102 in the placenta) [2]. Expression of $G H-1$ is controlled by the highly polymorphic proximal 103 promoter and by a locus control region placed $15-32 \mathrm{~kb}$ upstream of the gene that confers the 104 pituitary-specific, high-level expression of GH [18, 19].

$105 \mathrm{GH}-1$ consists of five exons and four introns (or intervening sequences; IVS), enabling 106 transcription of five known variants through alternative splicing $[1,20,21]$ (Fig.1 A). When $G H$ 1071 is correctly spliced, it produces the $22-\mathrm{kDa}$ isoform, which includes all five exons and represents the major biologically active form of $\mathrm{GH}$ present in the circulation. However, even 109 under normal condition, a small percentage of alternative splicing products is generated. These 110 splicing variants include:

(a) The $20-\mathrm{kDa}$ isoform (representing $5-10 \%$ of $\mathrm{GH}$ transcripts), as a result of the 112 activation of an in-frame cryptic splice site within exon 3 which causes deletion of amino acids 113 32-46;

114 (b) The $17.5-\mathrm{kDa}$ isoform, (representing $1-5 \%$ of $\mathrm{GH}$ transcripts) resulting from complete skipping of exon 3 with no biological activity; 
IGF-1 binding protein (IGFBP), mainly IGFBP-3, but also IGFBP-5, and the labile acid subunit (ALS) [29-32]. The 150-kDa ternary complex stabilizes IGF-1, prolonging its circulating half-life and regulating its availability to the target tissues [29].

IGF-1 has, in fact, growth stimulating effects on a wide variety of tissues but it has principally stimulatory effects on osteoblast and chondrocyte activity to promote growth for development during childhood and puberty. Further, GH normally regulates its own expression and production by both direct and indirect feedback (via IGF-1): IGF-1 leads to decreased secretion of GH by suppressing the somatotrophs and by stimulating release of somatostatin from the hypothalamus. In parallel, GH inhibits GHRH secretion and probably has a direct autocrine inhibitory effect on secretion from the somatotrophs [24].

\section{IGHD II}

IGHD II is the autosomal dominant form of IGHD and it is caused by heterozygous $G H-1$ mutations. In contrast to nonsense, missense and deletion mutations that are characteristic for the autosomal-recessive form of IGHD (IGHD I), the majority of IGHD II mutations described so far affect the splicing of $G H-1$ by different mechanisms $[5,6]$.

Splicing is the complex process by which exons are joined together to generate mature mRNA after removal of introns. It requires a complex interplay between cis- and trans-acting factors. In constitutive splicing, the cis-acting elements are the 5' splice site, branch point, polypyrimidine tract and the 3' splice site, each of which are defined by short, degenerate consensus sequences [33]. These elements are recognized by the spliceosome, a large macromolecular complex which 160 assembles onto them and catalyzes the splicing reaction in two transesterification steps [34]. However, often splice sites are weak and need additional sequences to be properly recognized. 
GH $[7,8]$. After being produced, the $17.5-\mathrm{kDa}$ isoform is detected by ER quality control mechanism and degraded via the proteasomal pathway. However, when the production rate of $17.5-\mathrm{kDa}$ isoform exceeds the proteasome degradation capacity, it accumulates in the cytoplasm leading to reduced cell proliferation and apoptosis of GC cells [47]. The amount of the $17.5-\mathrm{kDa}$ isoform has to reach a critical threshold to exert its dosage-dependent effect, with increasing amounts leading to reduced cell proliferation and apoptosis of somatotrophs [36].

Although it is clear that these mutant GH forms have an effect on the accumulation and secretion of GH, the exact cause of IGHD II is still unknown. Disrupting effects may occur at any stage during the secretory process.

\subsection{IGHD II animal models}

Additional insights about this disorder come from IGHD II animal models. Transgenic animals were generated by micro-nuclear injection of a fragment containing the locus control region of $G H-1$ and the genomic sequence of the IVS3 +1 GH mutation [7]. Three lines expressing $h G H-1$ transcript missing exon 3 in their pituitary cells with different relative copy numbers were established, giving phenotypes ranging from strongly to barely affected animals. The most severely affected line gave birth to mice that became progressively growth retarded compared to wild type littermates during early postnatal growth. They presented anterior pituitary hypoplasia, barely detectable levels of GH and multiple hormonal deficiencies and their pituitary was smaller than that of control mice due to the loss of somatotrophs. Their hypothalamic GHRH expression was upregulated and SRIF expression reduced consistent with their profound GHD. Few SVs were detected in the residual pituitary and somatotrophs were in turn invaded by macrophages. On the other hand, low copy number transgenic animals showed a mild effect with their body 


\subsection{IGHD II phenotype in patients and clinical implications}

Patients with IGHD II show substantial variation in their clinical phenotype. They present with low but detectable serum GH levels, variable height deficit and can show anterior pituitary hypoplasia on MRI $(38 \%, 50 \%)[4,48,49]$.

Reports on pedigrees with the Arg183His or E3+1G $>$ A mutations highlight the fact that patients with the same mutation can vary considerably in height $(\leq-4$ SDS to normal) and even attain normal adult height without treatment $[9,49]$.

Patients with the IVS3 +1 or IVS3 +2 splice site or the Pro89Leu mutations can develop additional pituitary hormone deficiencies, including ACTH, prolactin, TSH or gonadotropin deficiency [11, 50, 51]. The mechanism by which other endocrine axes are affected is not obvious, but might be through bystander damage from activated macrophages clearing dying somatotrophs, as observed in the transgene mouse study $[7,11]$.

Generally splice-site mutations, which exclusively produce the $17.5-\mathrm{kDa}$ isoform, cause, on average, an earlier age of onset and greater clinical severity [5], while ESE mutations cause a slightly less severe impact on patients height [38]. The quantitative and qualitative differences in the clinical phenotype are attributable to differences in the isoform ratio rather than the mutant transcript alone. Patients with the highest $17.5-\mathrm{kDa} / 22-\mathrm{kDa}$ ratio also have the lowest height standard deviation scores (SDS) and GH peak following pharmacological stimulation, while, on the contrary, those with the lowest $17.5-\mathrm{kDa} / 22-\mathrm{kDa}$ ratio can attain normal height despite never having been treated with rhGH [9]. 
In these patients, the GH-negative feedback on GHRH is reduced and it results in a chronic upregulation of GHRH and reduction of somatostatin expression. The constant upregulation of GHRH might increase the stimulatory drive on somatotrophs and the production of $\mathrm{GH}$, both from the normal and mutant allele, creating a vicious circle of increased levels of the $17.5-\mathrm{kDa}$ isoform that further accelerate damage to the anterior pituitary $[52,53]$. Interestingly, even in patients with a genetic cause (for example, Glu32Ala), IGHD seems to reverse when they are retested at the end of growth, in the transition period before transfer from pediatric to adult services, possibly because the treatment with rhGH removes the endogenous drive by GHRH and allows the recovery of somatotrophs. However, this effect is temporary, observed in patients tested at the time of transition, who should not be discharged from follow-up [39]. This evolving phenotype dictates the need for lifelong follow-up and possibly rhGH treatment in affected individuals [11]. Supplementation of rhGH therapy with somatostatin could be a valid option: it could increase the efficacy of GH therapy by inhibiting the production of endogenous GH and thereby preventing the production of the $17.5-\mathrm{kDa}$ isoform to progressively damage the remaining somatotrophs.

However, because IGHD II arises as a direct consequence of excessive production of a specific dominant-negative isoform, strategies designed to decrease the levels of the $17.5-\mathrm{kDa}$ isoform without affecting the normal $22-\mathrm{kDa}$ product may be useful to treat IGHD II patients with splicing mutations while avoiding the side-effects of long-term post-pubertal rhGH therapy.

\section{Rescue of IGHD II in vitro and in vivo by RNA interference (RNAi): a simple concept but} a complex reality

Over the last decade, double-stranded short interfering RNAs (siRNAs) have become a molecular 


\section{Viral-based therapy for IGHD II: background and progress in using viral vectors}

Gene therapy has been used successfully to increase protein level in vivo in several neurological disease models, including ALS, epilepsy and Parkinson's disease [59]. In the contest of IGHD II Lochmatter et al. tested a strategy involving lentiviral vector (LVs) expressing a human microRNA-30-based shRNA (shRNAmir) [60, 61], targeting the anomalous junction of exon 2 and exon 4 (shRNAmir- $\Delta 3$ ) of the $G H-1$ characterizing the misspliced 17.5-kDa isoform.

Using rat pituitary tumor GC cells expressing both human $w t-\mathrm{GH}$ and e17.5-kDa transcript, they demonstrated that shRNAmir efficiently reduces the expression of mutant protein, leading to an increase of $w t-\mathrm{GH}$ secretion, without affecting the viability of the transduced cells. Furthermore, cells expressing $17.5-\mathrm{kDa}$ mRNA upon doxycycline (DOX) induction transduced with shRNAmir- $\Delta 3$ significantly reduced the $17.5-\mathrm{kD}$ isoform at protein level and improved human $w t$-GH secretion in comparison to an shRNAmir targeting a scrambled sequence. Importantly, no toxicity due to shRNAmir expression could be observed in cell proliferation assays. Furthermore, confocal microscopy data strongly suggested that shRNAmir- $\Delta 3$ (exon 3 missing) enabled the recovery of GH granule storage and secretory capacity. Taken together, these viral vectors have shown their ability to stably integrate, express shRNAmir and rescue IGHD type II phenotype in rat pituitary cells. This methodology, although effective, raises specific safety and ethical issues. LVs are widely used as promising vehicles for their ability to provide long-term and stable gene expression and to infect non-dividing cells, such as neurons. Despite constant efforts are being made to improve safety and specificity, many concerns remain to be addressed including the possible generation of replication competent lentiviruses during vector production, mobilisation of the vector by endogenous retroviruses in the genomes of patients, insertional mutagenesis 
4346 fulfill this aim $[64,65]$.

347

HDACi are a relatively new group of epigenetic agents that have multiple substrates including histone and non-histone proteins, suggesting their involvement in multiple cellular processes. They alter the acetylation status of chromatin and other non-histone proteins, resulting in changes in gene expression. In normal cells (non-cancer cells), the response to HDACi depends on the cell type, on the structure and concentration of HDACi as well as the exposure time to HDAC inhibitors [64]. They are able to modify splicing by directly modifying the expression of specific splicing factors belonging to the serine/arginine (SR) rich protein.

SR proteins modulate selection of alternative splicing sites in a concentration-dependent and tissue-specific manner. They are part of a growing family of structurally related and highly conserved splicing factors characterized by the presence of 1-2 RNA recognition motifs (RRM) and by a different carboxyterminal domain that is highly enriched in Arg/Ser dipeptides (the RS domain). The RRMs mediate sequence-specific binding to RNA, determining in that way substrate specificity, whereas the RS domain seems to be involved mainly in protein-protein interactions. SR proteins that are bound to ESEs can promote exon definition by directly recruiting the splicing machinery through their RS domain and/or by antagonizing the action of nearby silencer elements. These two models of splicing enhancement are not necessarily mutually exclusive, as they might reflect different requirements in the context of different exons. Overexpression of splicing factors regulate the splicing pattern of alternatively spliced exons and differences in the levels of alternatively spliced transcripts were suggested to arise from differences in the relative levels of splicing factors (the SR and hnRNP families) [33].

This molecular approach was successfully tested for treatment of splicing disorders like cystic fibrosis (CF) and spinal muscular atrophy (SMA) [66-68]. 
SMA is an autosomal recessive neuromuscular disease characterized by degeneration of alpha

motor neurons in the spinal cord. It is caused by homozygous mutations of the survival motor neuron 1 (SMN1) gene. Normally, both gene copies (SMN1 and SMN2) are expressed, but they differ in the expression of full-length protein. $S M N 2$ gene preferentially gives rise to a truncated and less stable version of the SMN protein and thus can not compensate for SMN1 loss or mutations unless it is not present in multiple copies. The differences between these highly homologous genes are in their RNA expression patterns. Most SMN2 transcripts lack exons 3, 5, or most frequently, 7, with only a small amount of full-length mRNA generated. On the other hand, the SMN1 gene expresses mostly a full-length mRNA, and only a small fraction of its transcripts are spliced to remove exons 3, 5, or 7. The amount of exon 7-containing SMN protein has been shown to be an inverse indicator of disease severity in SMA patients and mice. Therefore, increasing the expression of intact SMN protein may have clinically therapeutic effects on SMA patients. Chang et. al. [66] showed that butyrate treatment of human SMA lymphoid cell lines increased the expression of exon 7-containing SMN protein from the SMN2 gene. After butyrate treatment in vitro and in vivo, the transcription pattern of SMN2 changed to an $S M N 1$-like transcription pattern, which was almost identical to the $S M N$ pattern in healthy individuals. Moreover, the clinical symptoms of the SMA-like mice improved after treatment with butyrate.

Following this initial report, Nissim-Rafinia et al, [67] restored the function of CFTR (cystic fibrosis transmembrane conductance regulator) channel: the ABC-transporter class ion-channel that transports chloride and thiocyanate across epithelial cell membranes. Mutations of the CFTR gene affect functioning of the chloride ion channels in epithelial cell membranes, leading to cystic fibrosis. The CFTR gene comprises 27 constitutively spliced exons, however several exons 
Upon butyrate treatment, GC-GHRHR cells coexpressing $w t$-hGH and each of the mutants displayed increased GH transcript level, intracellular GH content and GH secretion when compared with the corresponding untreated condition. The effect of butyrate was most likely mediated by the splicing factor ASF/SF2. Overexpression of ASF/SF2 in the same experimental setting promoted the amount of full-length transcripts and increased synthesis and secretion of the $22-\mathrm{kDa}$ isoform. Thus, it mirrors the effects of butyrate at the level of mRNA, protein and secretion.

Our results propose that, in the presence of splicing mutations, the $w t$-GH secretion can be rescued by increasing the expression of the $22-\mathrm{kDa}$ isoform. This might represent an alternative option to the down-regulation of the 17.5-kDa isoform as previously shown [56]. The knockdown of the $17.5-\mathrm{kDa}$ transcripts may reduce autosomal dominant effects and probably improve IGHD II condition. However, in an in vivo context, the reduced $w t$-GH transcript levels from a severely mutated allele can not be totally restored by RNAi, because shRNAmir acts by a posttranscriptional mechanism and does not reverse the mutant mRNAs into wt-molecules. Translation of the $17.5-\mathrm{kDa}$ mRNA is blocked, avoiding dominant-negative effect, but $w t-\mathrm{GH}$ expression and then secretion is overall diminished, because the mutant $\mathrm{GH}$ allele only synthesizes a small amount of functional $w t$-mRNAs.

Overall, these results provided direct evidence that changing the $17.5-\mathrm{kDa} / 22-\mathrm{kDa}$ ratio by increasing the level of 22-kDa transcripts can restore normal GH secretion and could represent a potential new and therapeutically beneficial approach to IGHD II.

\section{2 Benefits and drawbacks of small molecule as therapeutics}

Currently there are numerous classes of small molecules under investigation for their ability to 
contrast to siRNAs, AON survive and function inside the cell as a single strand [55].

In addition to their specificity, AONs are relatively non-invasive (they do not alter the genome directly) and very stable. They are efficiently and spontaneously internalized by cells in vivo, present high substrate specificity and low toxicity, and are not degraded by endogenous RNase $\mathrm{H}$.

The half-life of naked AON in mouse, human plasma and many mouse tissues is approximately 10-15 days [81]. Treatment of mice with a single injection of AON early in life has been shown to correct splicing and disease-associated phenotypes for up to a year [82, 83]. The basis of this longevity is unclear but may be attributable to the stability of AON in post-mitotic cells where they continue to influence splicing long after administration. Such phenomenon might be related to the ability of mammalian cells to accumulate systemically and locally administered singlestranded oligonucleotides and lipophilic-conjugated single- or double-stranded oligonucleotides inside the same subcellular compartment as the target RNA. The mechanisms for accumulation within cells are complex, but appear to involve novel endosomal transport mechanisms [81, 84]. The basic AON technology underwent over the years to different variations in order to provide additional functions. A number of different bifunctional AON have been designed. They are characterized by an antisense base pairing a sequence complementary to a consensus binding site sequence for a specific splicing factor. In this way, the AON can be directed to bind to the target RNA and at the same time to recruit a protein. This target protein, depending on its function and the location where the AON binds, can either enhance [85] or silence splicing[86, 87].

Because rescuing IGHD II by using AON has not been tested so far but has been successfully tested to promote exon inclusion in SMA[86], we believe that a good strategy for the future would be using, in combination with $\mathrm{HDACi}$, a chimeric antisense oligonucleotide comprising two parts: one which is complementary to the aberrantly spliced exon and provides exon 
4506 Since trans-splicing technology requires the delivery of DNA expression vectors to cells, the 
2

3

\section{Acknowledgements}

7521 This study was supported by a grant of Swiss National Science Foundation 320000-121998 to 8

922 PEM. 
[1] Mullis PE. Genetic control of growth. Eur J Endocrinol. 2005;152:11-31.

[2] Procter AM, Phillips JA, 3rd, Cooper DN. The molecular genetics of growth hormone deficiency. Hum Genet. 1998;103:255-72.

[3] Alatzoglou KS, Dattani MT. Genetic causes and treatment of isolated growth hormone deficiency-an update. Nat Rev Endocrinol. 2010;6:562-76.

[4] Alatzoglou KS, Turton JP, Kelberman D, Clayton PE, Mehta A, Buchanan C, et al. Expanding the spectrum of mutations in GH1 and GHRHR: genetic screening in a large cohort of patients with congenital isolated growth hormone deficiency. J Clin Endocrinol Metab. 2009;94:3191-9.

[5] Binder G, Keller E, Mix M, Massa GG, Stokvis-Brantsma WH, Wit JM, et al. Isolated GH deficiency with dominant inheritance: new mutations, new insights. J Clin Endocrinol Metab. 2001;86:3877-81.

[6] de Graaff LC, Argente J, Veenma DC, Herrebout MA, Friesema EC, Uitterlinden AG, et al. Genetic screening of a Dutch population with isolated GH deficiency (IGHD). Clin Endocrinol (Oxf). 2009;70:742-50.

[7] McGuinness L, Magoulas C, Sesay AK, Mathers K, Carmignac D, Manneville JB, et al. Autosomal dominant growth hormone deficiency disrupts secretory vesicles in vitro and in vivo in transgenic mice. Endocrinology. 2003;144:720-31.

[8] Salemi S, Yousefi S, Eble A, Deladoey J, Mullis PE. Impact of del32-71-GH (exon 3 skipped $\mathrm{GH})$ on intracellular GH distribution, secretion and cell viability: a quantitative confocal microscopy analysis. Horm Res. 2006;65:132-41. 
FIGURE LEGENDS 
4785 represented as ray-traced image with POVRAY (www.povray.org). The four $\alpha$-helices (I to IV)

7786 are orientated in an up-up-down-down conformation (Picture kindly provided by PD. Dr. Amit 8

9787 Pandey). 
GH-1 gene

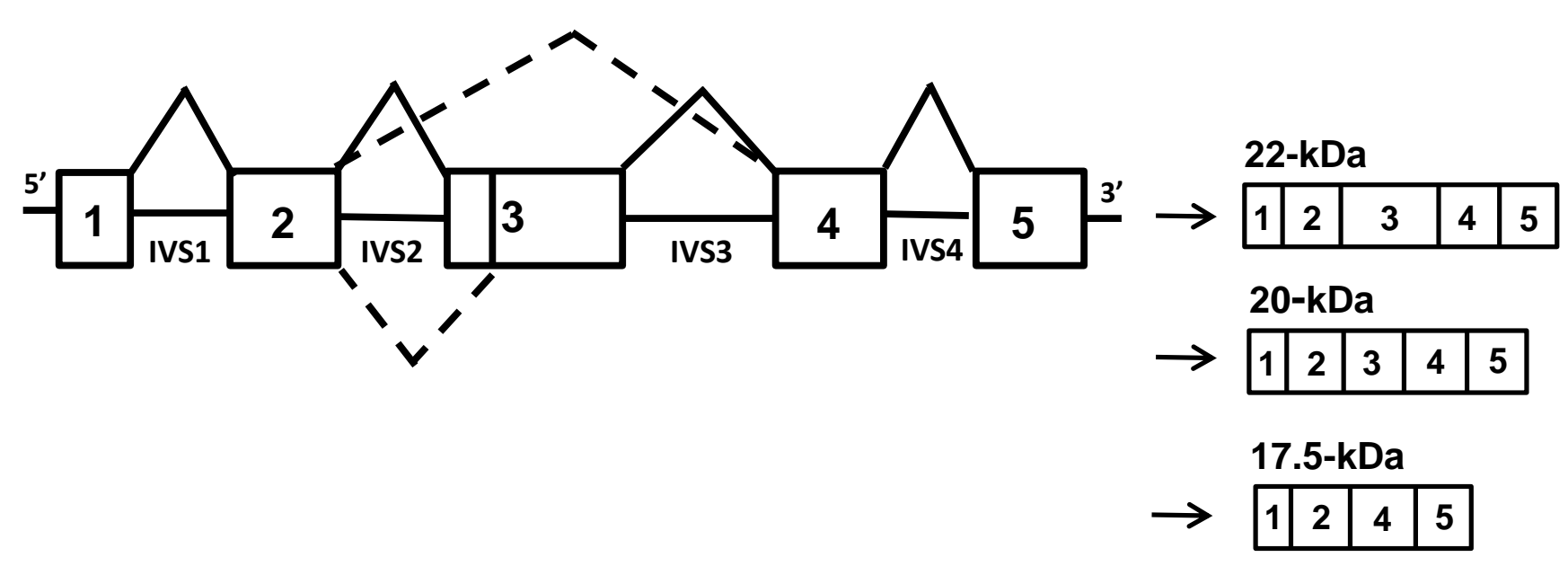

B

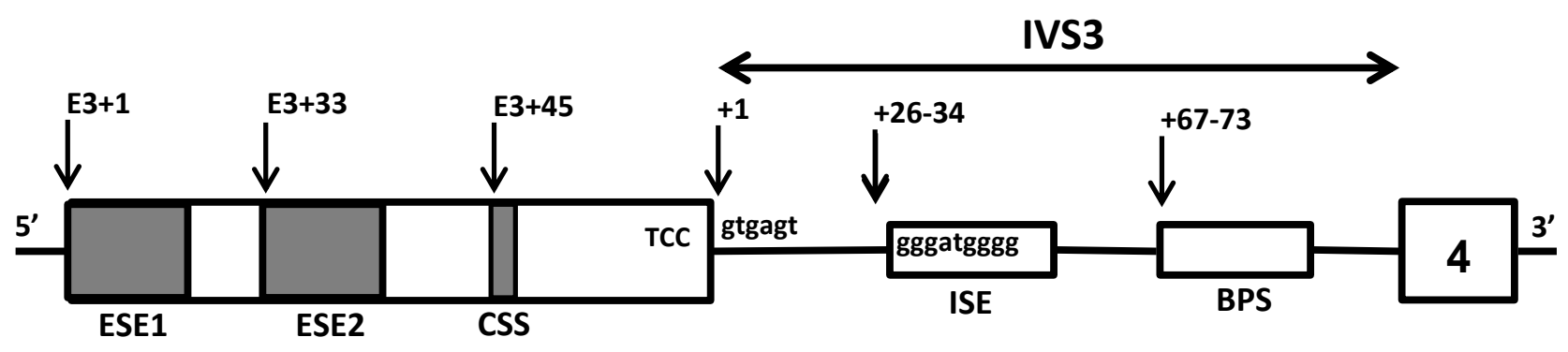

C

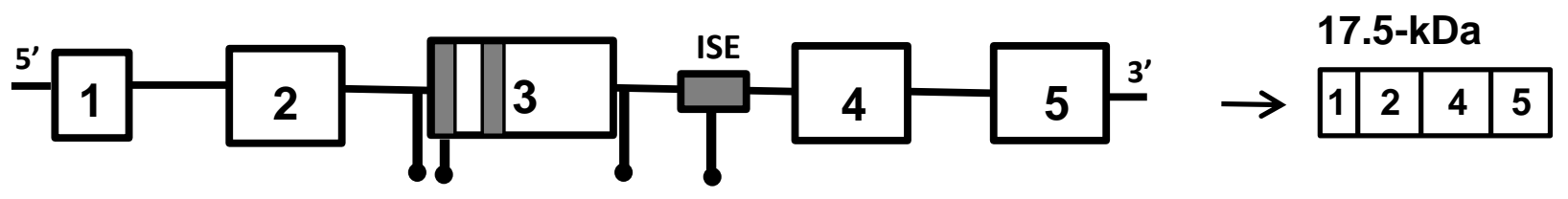




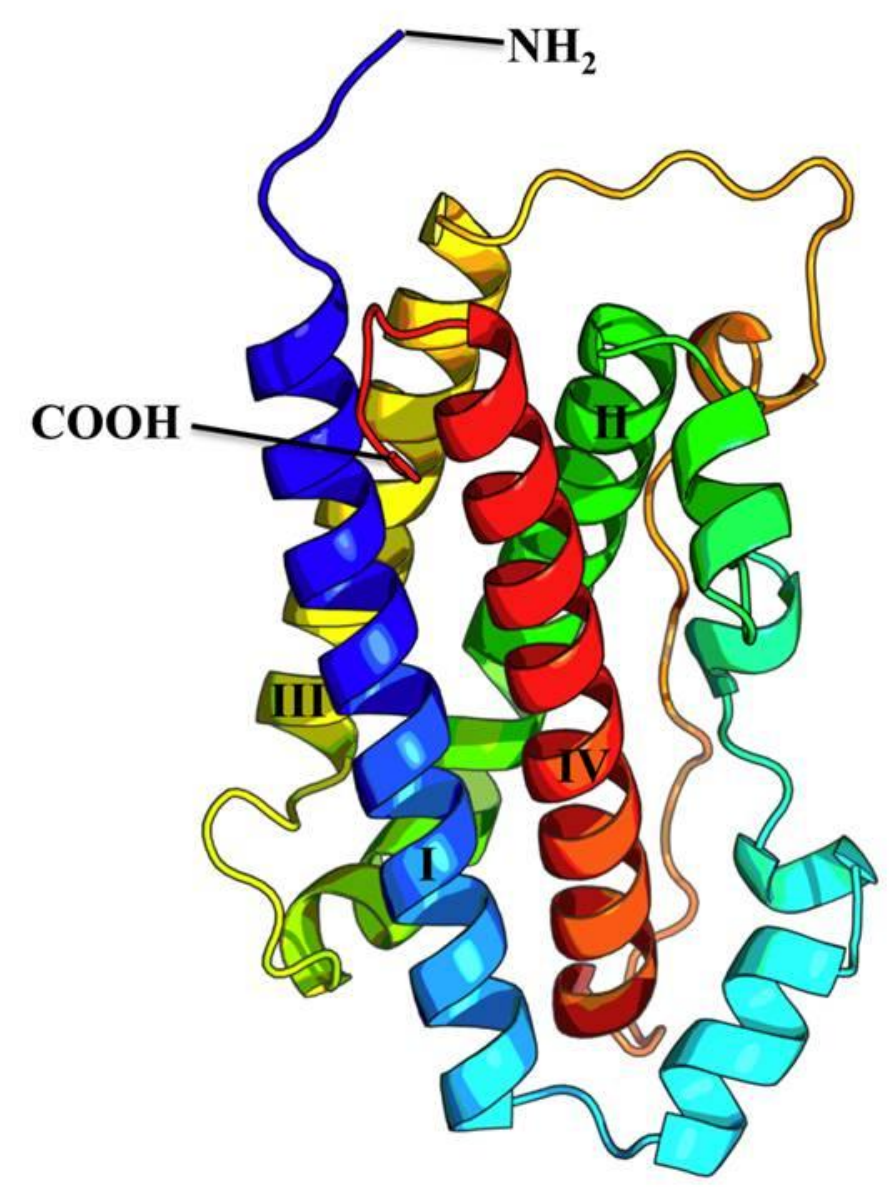


${ }^{\star}$ Graphical Abstract Growth Hormone Deficiency Type II

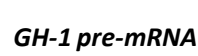

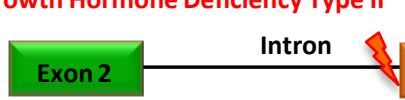

With treatment

GH-1 MRNA

Other GH-1 exon

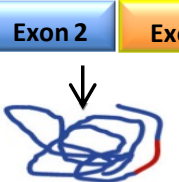

Exon 3

Exon 4

Full-length and biologically active 22-kDa GH isoform Normal GH secretion
Short and biologically inactive 17.5-kDa GH isoform

Reduced GH secretion

\section{Exon 4}

No treatment

Translation
Other GH-1 exon

\section{Exon 2}

Exon 3

Exon 4

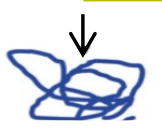

